\title{
Basophilic Focus
}

National Cancer Institute

\section{Source}

National Cancer Institute. Basophilic Focus. NCI Thesaurus. Code C138968.

A group of cells that exhibit some type of cytologic alteration resulting in basophilia, and that are confined to a specific area of the sample. 